\title{
ChemComm
}

\section{Angular dependence of the exchange interaction in fluoride-bridged $\mathrm{Gd}^{\mathrm{III}}-\mathrm{Cr}{ }^{\mathrm{III}}$ complexes ${ }^{\dagger}$}

Cite this: Chem. Commun., 2013, 49, 5583

Received 8th April 2013, Accepted 1st May 2013

DOI: $10.1039 / c 3 c c 42552 e$

www.rsc.org/chemcomm

The observed angular variation of the magnetic exchange coupling parameter in a series of fluoride-bridged chromium(III)-gadolinium(III) complexes is explained by DFT calculations.

The relatively new research field of magnetic refrigeration, by means of adiabatic demagnetisation, in paramagnetic, molecular, polynuclear, complexes ${ }^{1}$ and $\mathrm{MOFs}^{2}$ has reinvigorated the study of magnetically isotropic, polynuclear d- and f-metal ion compounds. ${ }^{3}$ As the magnetocaloric effect (MCE) delicately depends on the nature of the low-lying energy levels of such systems, it is necessary to develop methodologies for the prediction of the sign and magnitude of the magnetic exchange interactions between their constituent metal ions. ${ }^{4} \mathrm{Gd}^{\mathrm{III}}$ has a well-isolated, orbitally non-degenerate ${ }^{8} \mathrm{~S}_{7 / 2}$ ground term and can be considered magnetically isotropic. ${ }^{5}$ Similarly, $\mathrm{Cr}^{\mathrm{III}}$ has only weak magnetic anisotropy. ${ }^{6}$ Thus, a combination of these two metal-ions for the development of new molecular refrigerants seems promising. We have recently reported a pentanuclear, trigonal bipyramidal $\left\{\mathrm{Gd}_{3} \mathrm{Cr}_{2}\right\}$ complex displaying good refrigeration properties. ${ }^{7}$ We report here magneto-structural correlation studies on a simple dinuclear $\mathrm{Cr}^{\mathrm{III}}-\mathrm{Gd}^{\mathrm{III}}$ model complex and three, previously described, polynuclear $\mathrm{Gd}^{\mathrm{III}}-\mathrm{Cr}^{\mathrm{III}}$ complexes (Fig. 1). ${ }^{8}$ Characteristically, only very small differences in the $\mathrm{Cr}^{\mathrm{III}}-\mathrm{F}$ and $\mathrm{Gd}^{\mathrm{III}}-\mathrm{F}$ bond lengths are observed in the series 1-4 suggesting that the differences in exchange interactions are essentially governed by the $\mathrm{Gd}^{\mathrm{III}}-\mathrm{F}-\mathrm{Cr}^{\mathrm{III}}$ angle. The synthetic approach to the series is based on the kinetic robustness of $\mathrm{Cr}^{\mathrm{III}}$ which prevents the precipitation of highly insoluble $\mathrm{GdF}_{3} .{ }^{9}$ The intrinsic preference of the fluoride ion for linear bridging facilitates the prediction of the cluster topology,

\footnotetext{
${ }^{a}$ Department of Chemistry, Indian Institute of Technology Bombay, Powai, Mumbai, 400076, India. E-mail: rajaraman@chem.iitb.ac.in; Fax: +91-22-2576-7152, Tel: +91-22-2576-7183

${ }^{b}$ Department of Chemistry, University of Copenhagen, Universitetsparken 5, DK-2100 Copenhagen, Denmark. E-mail: bendix@kiku.dk; Fax: +45 35320214; Tel: +45 3520111 ${ }^{c}$ Institut Laue-Langevin, 38042 Grenoble Cedex 9, France

$\dagger$ Electronic supplementary information (ESI) available: Synthetic procedures, crystallographic details, spectroscopic results, reduced magnetization data, computational details. CCDC 921006. For ESI and crystallographic data in CIF or other electronic format see DOI: $10.1039 / \mathrm{c} 3 \mathrm{cc} 42552 \mathrm{e}$

$\ddagger$ These authors contributed equally to the work.
}
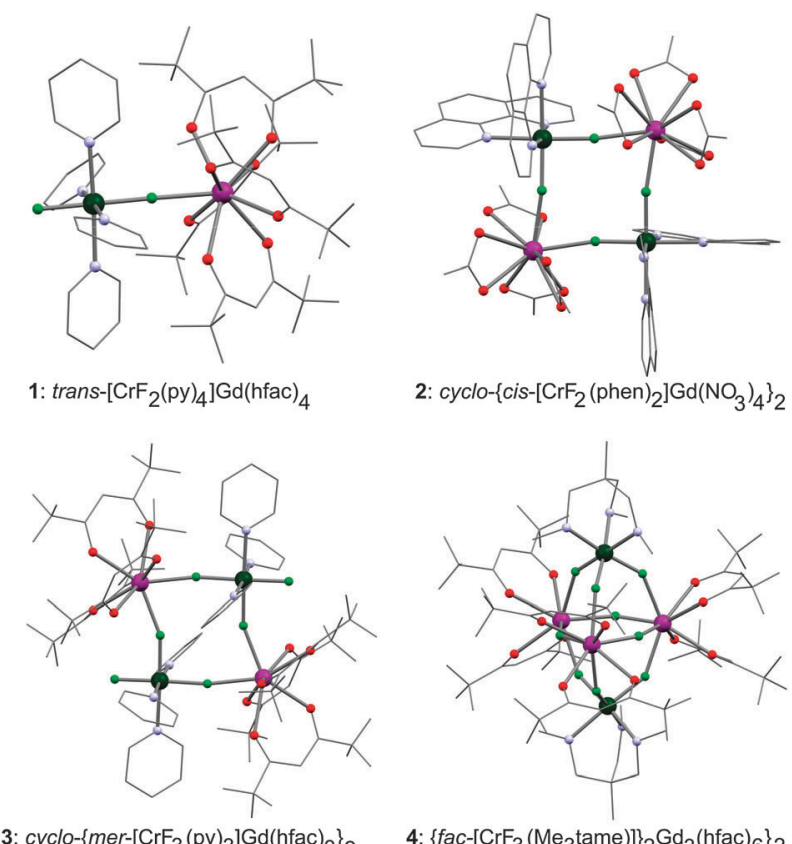

Fig. 1 Molecular structures of 1-4 (see also Scheme S1, ESIt). Gd, purple; Cr, dark green; $\mathrm{F}$, light green; $\mathrm{O}$, red; $\mathrm{N}$, blue. The auxiliary ligand sphere is shown as a wireframe and hydrogens are omitted for clarity.

giving rods for trans-difluorido, squares for cis-difluorido and trigonal bipyramids for fac-trifluorido chromium(III) precursors.

The $\chi T$ products of 1-4 are shown in Fig. 2 of which the magnetic properties of 2-4 have already been reported. ${ }^{7,8 b}$ The high-temperature $\chi T$ values are all in agreement with the values expected for the uncorrelated ions $\left(S_{\mathrm{Gd}}=7 / 2, S_{\mathrm{Cr}}=3 / 2, g=2.0\right.$ for both $\mathrm{Cr}^{\mathrm{III}}$ and $\mathrm{Gd}^{\mathrm{III}}$ ). On cooling, all $\chi T$ products decrease indicating the presence of intra-cluster antiferromagnetic interactions. All thermodynamic magnetometry data were fitted by use of the Levenberg-Marquardt algorithm ${ }^{10}$ and by numerical diagonalisation of the isotropic spin-Hamiltonian (1):

$$
\hat{H}_{\text {iso }}=\mu_{\mathrm{B}} \boldsymbol{B} \sum_{i} g_{i} \hat{\boldsymbol{S}}_{i}+J_{i j} \sum_{i, j>i} \hat{\boldsymbol{S}}_{i} \hat{\boldsymbol{S}}_{j}
$$



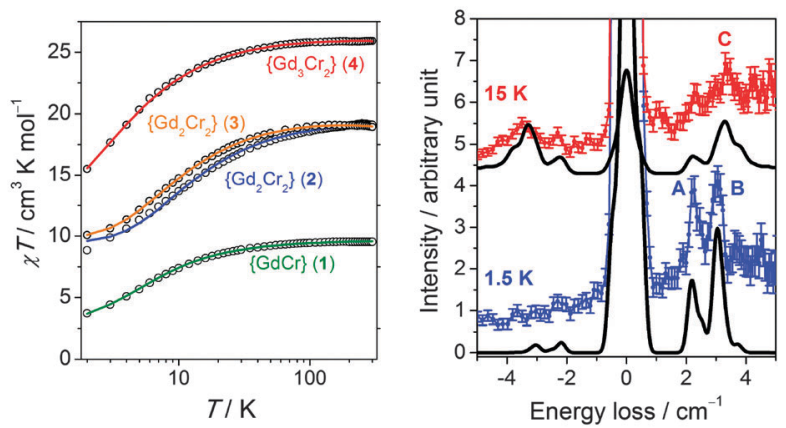

Fig. 2 (left) $\chi^{T}(\chi=M / H)$ data for 1-4 acquired with $H_{\mathrm{dc}}=1000$ Oe (circles) and best fits as described in the main text (solid lines). (right) INS spectra of 1 obtained with an incident neutron wavelength of $\lambda_{\mathrm{i}}=6.5 \AA$ at $T=1.5 \mathrm{~K}$ and $15 \mathrm{~K}$. Solid black lines are simulations.

where the indices $i$ and $j$ run through the constitutive single-ions of each compound, $g$ is the single-ion $g$-factor, $\hat{\boldsymbol{S}}$ is a spin-operator and $J$ is the isotropic exchange parameter. Note that a non-zero value for $J_{\mathrm{Gd}-\mathrm{Gd}}$ is only employed in the case of 4 . Independently fitting the $\chi T$ product and low temperature magnetization data (Fig. S2, ESI $\dagger$ ) of 1 affords $J=0.84(4) \mathrm{cm}^{-1}$ and $J=0.82(4) \mathrm{cm}^{-1}$, respectively, with the $g$ factors fixed to $g_{\mathrm{Cr}}=1.97$ and $g_{\mathrm{Gd}}=1.99$. Determination of an isotropic exchange parameter of this small magnitude solely from magnetic measurements may be inaccurate as other small terms, such as zero-field splittings, may be non-negligible, especially at low temperatures. Inelastic neutron scattering (INS) is a well-suited method for the determination of the magnetic anisotropy and exchange parameters in paramagnetic complexes due to the selection rules $\Delta S=0, \pm 1$ and $\Delta M_{S}=0, \pm 1$. Despite the extremely large neutron absorption cross-section of ${ }^{157} \mathrm{Gd}$ (ca. 16\% natural abundance), we managed to acquire INS spectra of 1 (Fig. 2 and Fig. S3, $\mathrm{ESI} \dagger$ ). Although noisy, the energy loss peaks A, at $2.2 \mathrm{~cm}^{-1}$, and B, at $3.0 \mathrm{~cm}^{-1}$, are clearly discernible in the spectrum at $\lambda_{\mathrm{i}}=6.5 \AA$ and assigned to magnetic excitations originating from the ground state, on the basis of their temperature dependence. Unfortunately, the poor quality of the observed $Q$-dependence, because of the presence of ${ }^{157} \mathrm{Gd}$ and large incoherent scattering from ${ }^{1} \mathrm{H}$, does not allow affirming the magnetic nature of these excitations. The experimental spectra were interpreted by use of home-written software ${ }^{11}$ invoking the anisotropic extension of spin-Hamiltonian (1) given in eqn (2):

$$
\hat{H}_{\text {aniso }}=\hat{H}_{\text {iso }}+D_{\mathrm{Cr}}\left(\hat{S}_{z, \mathrm{Cr}}^{2}-\frac{1}{3} S_{\mathrm{Cr}}\left(S_{\mathrm{Cr}}+1\right)\right)
$$

where $D_{\mathrm{Gd}}$ is neglected. ${ }^{5}$ For comparison, the broader, but more intense, spectrum at $\lambda_{\mathrm{i}}=4.8 \AA$ is shown in Fig. S3 (ESI $\dagger$ ). In the $T=15 \mathrm{~K}$ spectrum (Fig. 2, right), a hot peak (C) arises at $3.3 \mathrm{~cm}^{-1}$. For $D_{\mathrm{Cr}}=0$, the $S=3,4$ and 5 spin manifolds are separated from the $S=2$ ground state by $3 J, 7 J$ and $12 J$ (Fig. S4, ESI $)$ ), respectively, and only one prominent INS peak would be observed at low temperature from the $S=2$ to $S=3$ excitation (Fig. S5, ESI + ). Introduction of $D_{\mathrm{Cr}}$ breaks the degeneracy of the $M_{S}$ levels, and an optimized value of $D_{\mathrm{Cr}}=0.5 \mathrm{~cm}^{-1}$, together with $J=0.87 \mathrm{~cm}^{-1}$, results in good agreement with the experiment (cf. Fig. 2, right).

The obtained $J$ parameters for $\mathbf{2 - 4}$, for which the INS spectra were not acquired, are shown together with that for $\mathbf{1}$ in Fig. 3 as
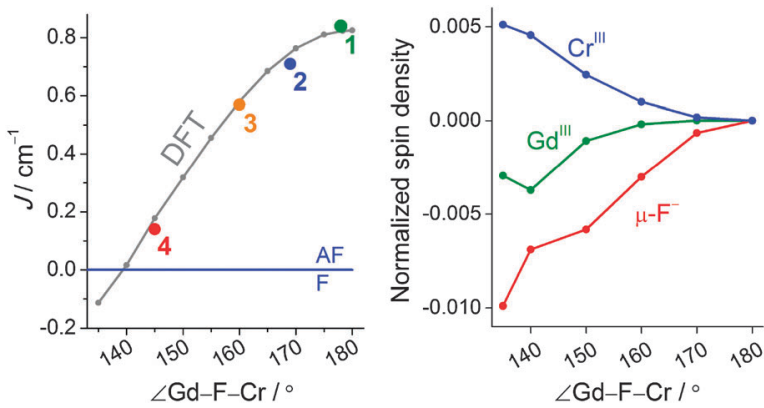

Fig. 3 (left) Angular dependence on the J parameters for 1-4 (coloured circles) and DFT results (grey circles). (right) Normalized spin density plot for an angular distortion of 1

a function of the Gd-F-Cr angle. All the extracted couplings are antiferromagnetic and their strength seems to correlate with the $\mathrm{Gd}-\mathrm{F}-\mathrm{Cr}$ angle. Magnetic interactions in $\{3 \mathrm{~d}-\mathrm{Gd}\}$ dinuclear complexes are in general ferromagnetic with some notable exceptions, such as a cyanide-bridged $\{\mathrm{CrGd}\}$ complex. ${ }^{12,13} \mathrm{~A}\left\{\mathrm{GdCr}_{4}\right\}$ hydroxide bridged complex is also reported to be antiferromagnetic in nature. ${ }^{14}$ This fuelled our curiosity as to whether $\mathrm{Cr}^{\mathrm{III}}$ in particular promotes antiferromagnetic interactions. Indeed, for 1-4, the exchange interaction is antiferromagnetic. Moreover, there is a structural correlation to this antiferromagnetic interaction as shown in Fig. 3, which presents $J$ as a function of the Gd-F-Cr angle. To shed light on this issue, as well as on the angular dependence of $J$, we performed DFT (B3LYP/TZV) calculations on 1. All the calculations were performed with the Gaussian 09 program suite ( $c f$. ESI $\dagger$ for computational details). The computed $J$ value of $0.80 \mathrm{~cm}^{-1}$ for $\mathbf{1}$ is in excellent agreement with the values extracted from experiments (see Table S2 for DFT computed energies, ESI $\dagger$ ). The reproduction of both the sign and the magnitude of $J$ provides confidence in the computed $J$ values.

Understanding the mechanism by which the coupling is operational in this $\{\mathrm{Cr}-\mathrm{Gd}\}$ pair is important, as it is expected to provide clues about the nature of the exchange interaction and its angular dependence. The following generic mechanistic points emerge from our earlier studies. ${ }^{15 a-f}$ In a general $\{3 \mathrm{~d}-4 \mathrm{f}\}$ pair, the overlap between the $4 \mathrm{f}$ orbitals and the $3 \mathrm{~d}$ orbitals only partly determines the nature of the magnetic exchange interaction. Orthogonality between the $3 \mathrm{~d}$ and $4 \mathrm{f}$ orbitals results in ferromagnetic, $J_{\mathrm{F}}$, contributions, whereas, non-vanishing overlap of the same orbitals results in antiferromagnetic, $J_{\mathrm{AF}}$, contributions to the magnetic exchange. Additionally, charge transfer excitations from a 3d orbital to an empty 5d orbital of the lanthanide contribute to $J_{\mathrm{F}}$. Since the $4 \mathrm{f}$ orbitals are contracted, contributions to $J_{\mathrm{AF}}$ are generally weak and therefore most of the $\{3 \mathrm{~d}-4 \mathrm{f}\}$ pairs exhibit ferromagnetic behaviour. In complex $\mathbf{1}$, the unpaired electrons on $\mathrm{Cr}^{\mathrm{III}}$ are located in the $\mathrm{t}_{2 \mathrm{~g}}\left\{\mathrm{~d}_{x y}, \mathrm{~d}_{x z}, \mathrm{~d}_{y z}\right\}$ set of orbitals. Since these orbitals exhibit $\pi$ character, an efficient $\sigma$-type charge transfer from $3 \mathrm{~d}$ to $5 \mathrm{~d}$, observed for $\left\{\mathrm{Cu}^{\mathrm{II}}\left(\mathrm{t}_{2 \mathrm{~g}}{ }^{6} \mathrm{e}_{\mathrm{g}}{ }^{3}\right)-\mathrm{Gd}\right\}$ and $\left\{\mathrm{Ni}\left(\mathrm{t}_{2 \mathrm{~g}}{ }^{6} \mathrm{e}_{\mathrm{g}}{ }^{2}\right)-\mathrm{Gd}\right\}$ complexes, is absent in $\mathbf{1}$ and thus, the $J_{\mathrm{F}}$ contribution is significantly reduced. ${ }^{15 a-c}$ To estimate the $J_{\mathrm{AF}}$ contributions, the overlap integrals $\left(S_{\mathrm{ab}}\right)$ between the magnetic orbitals of $\mathrm{Cr}^{\mathrm{III}}$ and $\mathrm{Gd}^{\mathrm{III}}$ have been computed and significant $S_{\mathrm{ab}}$ values have been detected indicating a relatively strong $3 \mathrm{~d}-4 \mathrm{f}$ overlap (Fig. S6, ESI †). Previously, we have shown that the number 


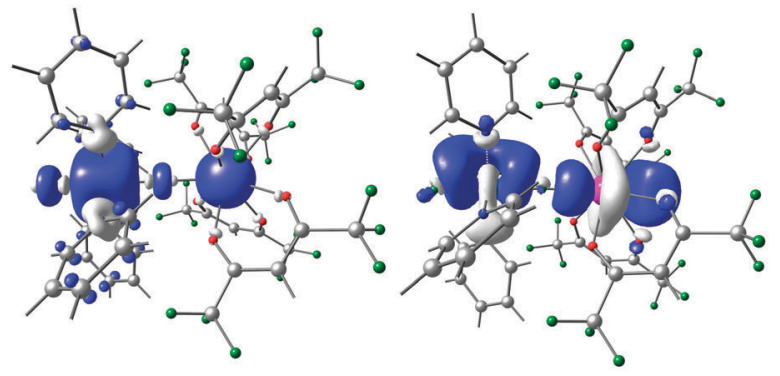

Fig. 4 (left) Spin density plot for the parent $\mathrm{Cr}$-Gd complex. (right) Representation of charge transfer donor(Cr'II $\left.3 d_{z^{2}}\right)$-acceptor $\left(G d^{\prime \prime \prime} 5 d_{z^{2}}\right)$ NBO plot from second order perturbation theory analysis.

of bridges plays a vital role in the sign of magnetic coupling. In particular, when going from two alkoxo-bridges to one bridge in $\{3 \mathrm{~d}-\mathrm{Gd}\}$ complexes, a strong antiferromagnetic interaction is predicted, essentially due to a larger direct 3d-4f orbital overlap and thus a large $J_{\mathrm{AF}}$ contribution. ${ }^{15 b}$ This correlates well with the single fluoride bridge and the antiferromagnetic interaction observed in this series. Additional insight into the mechanism of the exchange interaction is provided by DFT-calculated spin densities. Spin densities of 3.09 on $\mathrm{Cr}^{\mathrm{III}}$ and $7.03 \mathrm{Gd}^{\mathrm{III}}$ indicate predominant spin polarisation (Fig. 4, left; Table S3, ESI $\dagger$ ). All the coordinated nitrogen and oxygen atoms have negative spin densities, however the fluoride ions, both the bridging and the terminal, have positive spin densities in the high spin state ( $c f$. Table S4, ESI $\dagger$ ). The bridging fluoride has negative spin density values in the broken symmetry state and this essentially indicates that delocalization from $\mathrm{Cr}^{\mathrm{III}}$ predominates over the $\mathrm{Gd}^{\mathrm{III}}$. Comparing the magnitude and the sign of the spin densities at the fluoride bridge, it is apparent that a mixture of spin delocalization and polarization is operational on these atoms and this diminishes the charge transfer component and thus reduces the $J_{\mathrm{F}}$ part. This is also supported by our NBO analysis where an extremely weak $\mathrm{Cr}^{\mathrm{III}} 3 \mathrm{~d}_{z^{2}}-\mathrm{Gd}^{\mathrm{III}} 5 \mathrm{~d}_{z^{2}}$ donor-acceptor interaction has been detected (Fig. 4, right). To gain insight into the angle dependency, the $\mathrm{Cr}-\mathrm{F}-\mathrm{Gd}$ angle was gradually varied from $135^{\circ}$ to $180^{\circ}$ in steps of $10^{\circ}$. The computed variation of $J$ with the bridging angle is shown in Fig. 3 along with the experimental points.

As the angle increases the $J$ becomes more antiferromagnetic, but shows a plateau at larger angles. On the other hand at an acute angle (at $c a .138^{\circ}$ ), a switch from antiferro- to ferromagnetic interaction is predicted. Mapping the experimental $J$ s and structural parameters on the computed graph yields an excellent match. The plateau at higher angles and less antiferromagnetic $J$ at lower angles was nicely reproduced. To analyse the factors behind the observed trend, we have computed the overlap integrals, which reveal that there is a significant decrease in the $\mathrm{Cr}-\mathrm{Gd}$ orbital overlap as the angle decreases leading to a reduction in the $J_{\mathrm{AF}}$ contribution. ( $c f$. Table S5, ESI $\dagger$ ) This along with a moderate increase in the charge transfer (cf. 5d occupation in Table S6, ESI $\dagger$ ) leads to a predicted ferromagnetic coupling at lower $\mathrm{Cr}-\mathrm{F}-\mathrm{Gd}$ angles. Our analysis reveals that at lower angles, the metal ion spin densities increase and, concomitantly, for $\mu-\mathrm{F}^{-}$the spin densities decrease proportionally indicating an increasing ferromagnetic contribution as evidenced from the computed $J$ values (see Fig. 3, right; Table S4, ESI $\dagger$ ).
In conclusion, the angular dependence of the exchange in a small family of fluoride-bridged $\left\{\mathrm{Gd}^{\mathrm{III}}{ }_{x} \mathrm{Cr}^{\mathrm{III}}{ }_{y}\right\}$ complexes has been analysed. The observed variation and magnitude of $J$ is reproduced and rationalised by DFT calculations in terms of geometric variation of $3 \mathrm{~d}-4 \mathrm{f}$ overlap and charge transfer. This insight paves the way for preparative control of magnetic exchange interactions in systems with small magnetic anisotropy and thereby for the development of new molecular, magnetic coolers.

K.S.P. and S.P. thank the Danish Ministry of Science, Innovation and Higher Education for an EliteForsk travel scholarship and a Sapere Aude Fellowship (10-081659), respectively. GR thanks DST (SR/S1/IC-41/2010; SR/NM/NS-1119/2011) for financial support and SKS thanks IITB for SRF fellowship. GR and SKS acknowledge the HPC facility at IITB.

\section{Notes and references}

1 (a) M. Evangelisti and E. K. Brechin, Dalton Trans., 2010, 39, 4672-4676; (b) J. W. Sharples and D. Collison, Polyhedron, 2013, 54, 91-103.

2 (a) G. Lorusso, M. A. Palacios, G. S. Nichol, E. K. Brechin, O. Roubeau and M. Evangelisti, Chem. Commun., 2012, 48, 7592-7594; (b) R. Sibille, T. Mazet, B. Malaman and M. François, Chem.-Eur. J., 2012, 18, 12970.

3 (a) R. E. P. Winpenny, Chem. Soc. Rev., 1998, 27, 447-452; (b) C. Benelli and D. Gatteschi, Chem. Rev., 2002, 102, 2369-2388; (c) M. Andruh, J.-P. Costes, C. Diaz and S. Gao, Inorg. Chem., 2009, 48, 3342-3359.

4 E. Cremades, S. Gómez-Coca, D. Aravena, S. Alvarez and E. Ruiz, J. Am. Chem. Soc., 2012, 134, 10532-10542.

5 O. Kahn, Molecular Magnetism, VCH, New York, 1993.

6 H. I. Karunadasa, K. D. Arquero, L. A. Berben and J. R. Long, Inorg. Chem., 2010, 49, 4738-4740.

7 T. Birk, K. S. Pedersen, C. Aa. Thuesen, T. Weyhermüller, M. SchauMagnussen, S. Piligkos, H. Weihe, S. Mossin, M. Evangelisti and J. Bendix, Inorg. Chem., 2012, 51, 5435-5443.

8 (a) J. Dreiser, K. S. Pedersen, C. Piamonteze, S. Rusponi, Z. Salman, Md. E. Ali, M. Schau-Magnussen, C. Aa. Thuesen, S. Piligkos, H. Weihe, H. Mutka, O. Waldmann, P. Oppeneer, J. Bendix, F. Nolting and H. Brune, Chem. Sci., 2012, 3, 1024-1032; (b) C. Aa. Thuesen, K. S. Pedersen, M. Schau-Magnussen, M. Evangelisti, J. Vibenholt, S. Piligkos, H. Weihe and J. Bendix, Dalton Trans., 2012, 41, 11284-11292.

9 (a) A. McRobbie, A. R. Sarwar, S. Yeninas, H. Nowell, M. L. Baker, D. Allan, M. Luban, C. A. Muryn, R. G. Pritchard, R. Prozorov, G. Timco, F. Tuna, G. F. S. Whitehead and R. E. P. Winpenny, Chem. Commun., 2011, 47, 6251-6253; (b) J. Dreiser, K. S. Pedersen, T. Birk, M. Schau-Magnussen, C. Piamonteze, S. Rusponi, T. Weyhermüller, H. Brune, F. Nolting and J. Bendix, J. Phys. Chem. A, 2012, 116, 7842-7847.

10 W. H. Press, S. A. Teukolsky, W. T. Vetterling and B. P. Flannery, Numerical Recipes in C: The Art of Scientific Computing, Cambridge University Press, Cambridge, MA, 2nd edn, 1992.

11 P. L. W. Tregenna-Piggott, D. Sheptyakov, L. Keller, S. I. Klokishner, S. M. Ostrovsky, A. V. Palii, O. S. Reu, J. Bendix, T. Brock-Nannestad, K. Pedersen, H. Weihe and H. Mutka, Inorg. Chem., 2009, 48, 128-137.

12 (a) N. Georgopoulou, R. Adam, C. P. Raptopoulou, V. Psycharis, R. Ballesteros, B. Abarca and A. K. Boudalis, Dalton Trans., 2010, 39, 5020-5027; (b) M. Ferbinteanu, F. Cimpoesu, M. A. Gîrțu, C. Enachescu and S. Tanase, Inorg. Chem., 2012, 51, 40-50.

13 H.-Z. Kou, S. Gao, C.-H. Li, D.-Z. Liao, B.-C. Zhou, R.-J. Wang and Y. Li, Inorg. Chem., 2002, 41, 4756-4762.

14 D. J. Hodgson, K. Michelsen and E. J. Pedersen, J. Chem. Soc., Chem. Commun., 1988, 1558-1559.

15 (a) S. K. Singh, N. K. Tibrewal and G. Rajaraman, Dalton Trans., 2011, 40, 10897-10906; (b) S. K. Singh and G. Rajaraman, Dalton Trans., 2013, 42, 3623-3630; (c) G. Rajaraman, F. Totti, A. Bencini, A. Caneschi, R. Sessoli and D. Gatteschi, Dalton Trans., 2009, 3153-3161; (d) J. Paulovic, F. Cimpoesu, M. Ferbinteanu and K. Hirao, J. Am. Chem. Soc., 2004, 126, 3321-3331; (e) T. Rajeshkumar and G. Rajaraman, Chem. Commun., 2012, 48, 7856-7858; $(f)$ S. K. Singh, T. Rajeshkumar, V. Chandrasekhar and G. Rajaraman, Polyhedron, 2013, DOI: 10.1016/j.poly.2013.02.037. 